\title{
OPTIMIZATION OF THIN SHELL STRUCTURES USING FSD ALGORITHMS
}

The paper presents a theoretical and numerical study of the efficiency of the fully stress design (FSD) algorithm in the case of thin shell finite elements. Relation between membrane forces, bending moments and the element thickness is analysed by means of numerical tests. Subsequent numerical testing and a new iterative algoritm to providing the rapid convergence of the optimizing process is proposed.

Keywords: stress analysis, thin shell finite element, fully stress design

\section{Introduction}

Structural optimization and development of new optimizing methods have been topical problems for several decades. At the present time the current issues are problems which have global character such as ecology, energetic sources, mineral and natural wealth, effectiveness of production and so on. Optimization process is used in all fields participating in development or innovation of technical devices and solutions. At the present time optimization and innovation are inseparable parts of every existing or developed engineering work analysed by means of computational technique [1].

Expansion of computational technique allowed putting qualitatively new approaches in designing machines and appliances into practice. The problem of proper designing and constructing of machines gets new dimensions and wide scope for solving other unsolved problems by establishing computers and consequent creating and developing corresponding software. An optimized design is comprehended as a technically realizable design of structure which is the best from all possible designs for a given goal [2].

Optimization of mechanical systems combines numerical mathematics and engineering mechanics. It is used in applications in civil engineering, mechanical engineering, automotive and ship-building industry, and so on. It made the biggest progress in last thirty years thanks to utilizing very fast numerical computers and computer graphics. When choosing cost, weight of structure or maximum power at a limited cost as a design criterion, the importance of optimization is evident.

The main task for a designer is still to propose dimensions of the structure properly with respect to its minimal weight, proper geometry, or some dynamical properties. The main goal is to spare material and find the best solution from a constructional point of view as well as the view of utilization of material and technologies [1].

The effort to create an optimal structure is not new, of course, but using optimization approaches in practical work of a designer can be achieved only by utilizing numerical methods and a powerful computational technique. Numerical methods are forcing out analytical methods which are time-consuming and improper for a lot of practical problems [3, 4].

Today we expect that designed objects will be optimally balanced in term of entire life cycle, i.e. projecting, manufacturing, running, maintaining and liquidating. The mentioned process relates mainly with economic aspects of each stage. Achieving this goal is very difficult, because a designer is usually met with contradictory demands related to individual stages of the mentioned life cycle of a designed object [4].

First formulations of optimization problems in the form of mathematical programming have been occurring approximately since1960. One of the pioneers who significantly influenced the development of the optimal designing of constructions of machines and their components, was undoubtedly Schmit. He linked optimization methods with a new and progressive computational method at that time - the finite element method as one of the first. At that time, the weight of a monitored object or some strength condition was the objective function. Optimization process was gradually improving by adding other limiting conditions. In the second half of the last century other works of similar nature, which extended options in the field of optimal designing of parameters of machines and their components into automated approaches occurred. We can not omit works of Kirch, Morrow, or Gallagher. Plenty of effective approaches were designed. They were based not only on a purely mathematical comprehension of optimization problem, but also a little bit non-traditional or unaccustomed appro-

\footnotetext{
* Milan Saga, Martin Dudinsky, Peter Pechac

Department of Applied Mechanics, Faculty of Mechanical Engineering, University of Zilina, Slovakia, E-mail: milan.saga@fstroj.uniza.sk
} 
aches which play an important role in solving various technical problems were utilized. These approaches use some of the basic principles of mechanics. For example, the method which is known as the fully stress design (FSD), originated from the idea of independence of axial forces in statically determinate truss structures. Its application is useful mainly thanks to its effectiveness. However, it is limited only to problems of strength dimensioning and it turned out to be a certain disadvantage in creating universal program systems. In this article theory of FSD will be described and applied specially for thin shell structures in spite of its lower universality [2, 5].

\section{Stress Calculation of thin Shellfinite Element}

We will focus on well-known shell finite elements (Kirchhoff's or Mindlin's formulation) [1, 6, 7, 8], mainly on the stress calculation. The stiffness parameters depend on material constants and element geometry, mainly on its thickness. The stress calculation process is based on the expression of the $j$-th element membrane forces and bending moments (without shear forces) [6,9], i.e.

$$
\begin{aligned}
& {\left[\begin{array}{lll}
F_{x x} & F_{y y} & F_{x y}
\end{array}\right]_{j}^{T}=\mathbf{F}_{m}^{j}=\int_{S} \mathbf{E}_{m}^{j} \cdot \boldsymbol{\varepsilon}_{m}^{j} d S_{j}=} \\
& =\mathbf{E}_{m}^{j} \cdot \int_{S} \mathbf{B}_{m}^{j} d S_{j} \cdot \mathbf{u}_{L}^{j}=t_{j} \cdot \mathbf{D}_{j} \cdot \mathbf{I}_{b}^{j} \cdot \mathbf{u}_{L}^{j}
\end{aligned}
$$

and

$$
\begin{aligned}
& {\left[\begin{array}{lll}
M_{x x} & M_{y y} & M_{x y}
\end{array}\right]_{j}^{T}=\mathbf{M}_{b}^{j}=\int_{S} \mathbf{E}_{b}^{j} \cdot \varepsilon_{b}^{j} d S_{j}=} \\
& =\mathbf{E}_{b}^{j} \cdot \int_{S} \mathbf{B}_{b}^{j} \cdot d S_{j} \cdot \mathbf{u}_{L}^{j}=\frac{t_{j}^{3}}{12} \cdot \mathbf{D}_{j} \cdot \mathbf{I}_{b}^{j} \cdot \mathbf{u}_{L}^{j}
\end{aligned}
$$

The auxiliary matrices $\mathbf{I}_{m}$ and $\mathbf{I}_{b}$ can be calculated only using the numerical approach. Further details about $\mathbf{E}_{m}, \mathbf{E}_{b}, \mathbf{D}, \mathbf{B}_{m}, \mathbf{B}_{b}$, $\mathbf{u}_{e l}$ and $t$ are presented in [9]. The extreme stress values can be expected at the top or at the bottom surface. Generally, it means

$$
\begin{aligned}
& {\left[\sigma_{m b}\right]_{t o p}^{j}=\left\{\begin{array}{l}
\sigma_{x x} \\
\sigma_{y y}^{j} \\
\sigma_{x y}
\end{array}\right\}_{t o p}=\left[\begin{array}{cccccc}
1 / t_{j} & 0 & 0 & 6 / t_{j}^{2} & 0 & 0 \\
0 & 1 / t_{j} & 0 & 0 & 6 / t_{j}^{2} & 0 \\
0 & 0 & 1 / t_{j} & 0 & 0 & 6 / t_{j}^{2}
\end{array}\right] .} \\
& \left\{\begin{array}{l}
F_{x x} \\
F_{y y} \\
F_{x y} \\
M_{x x} \\
M_{y y} \\
M_{x y}
\end{array}\right\}=[\mathbf{A}]_{t o p}^{j} \cdot \mathbf{f}^{j}
\end{aligned}
$$

for top element surface and

$$
\begin{aligned}
& {\left[\sigma_{m b}\right]_{b o t}^{j}=\left\{\begin{array}{l}
\sigma_{x x} \\
\sigma_{y y}^{j} \\
\sigma_{x y}
\end{array}\right\}_{b o t}=\left[\begin{array}{cccccc}
1 / t_{j} & 0 & 0 & -6 / t_{j}^{2} & 0 & 0 \\
0 & 1 / t_{j} & 0 & 0 & -6 / t_{j}^{2} & 0 \\
0 & 0 & 1 / t_{j} & 0 & 0 & -6 / t_{j}^{2}
\end{array}\right]} \\
& \left\{\begin{array}{l}
F_{x x} \\
F_{y y} \\
F_{x y} \\
M_{x x} \\
M_{y y} \\
M_{x y}
\end{array}\right\}=[\mathbf{A}]_{b o t}^{j} \cdot \mathbf{f}^{j}
\end{aligned}
$$

for the bottom element surface. Generally, the "top" or "bottom" von Mises stresses may be calculated from relations [10]

$$
\begin{aligned}
& \sigma_{\text {eqtop }}^{2}=\left[\boldsymbol{\sigma}_{m b}\right]_{\text {top }}^{T} \cdot \mathbf{T}_{m b} \cdot\left[\boldsymbol{\sigma}_{m b}\right]_{\text {top }}=\mathbf{f}^{T} \cdot[\mathbf{A}]_{\text {top }}^{T} \cdot \\
& \cdot \mathbf{T}_{m b} \cdot[\mathbf{A}]_{\text {top }} \cdot \mathbf{f}=\mathbf{f}^{T} \cdot \mathbf{T}_{\text {top }} \cdot \mathbf{f}
\end{aligned}
$$

or

$$
\begin{aligned}
& \sigma_{e q \mid b o t}^{2}=\left[\boldsymbol{\sigma}_{m b}\right]_{b o t}^{T} \cdot \mathbf{T}_{m b} \cdot\left[\boldsymbol{\sigma}_{m b}\right]_{b o t}=\mathbf{f}^{T} \cdot[\mathbf{A}]_{b o t}^{T} \cdot \\
& \cdot \mathbf{T}_{m b} \cdot[\mathbf{A}]_{b o t} \cdot \mathbf{f}=\mathbf{f}^{T} \cdot \mathbf{T}_{b o t} \cdot \mathbf{f}
\end{aligned}
$$

where

$$
\begin{aligned}
\mathbf{T}_{m b} & =\left[\begin{array}{ccc}
1 & -0.5 & 0 \\
-0.5 & 1 & 0 \\
0 & 0 & 3
\end{array}\right], \\
\mathbf{T}_{t o p} & =\left[\begin{array}{cccccc}
1 / t^{2} & -1 / 2 t^{2} & 0 & 6 / t^{3} & -3 / t^{3} & 0 \\
-1 / 2 t^{2} & 1 / t^{2} & 0 & -3 / t^{3} & 6 / t^{3} & 0 \\
0 & 0 & 3 / t^{2} & 0 & 0 & 18 / t^{3} \\
6 / t^{3} & -3 / t^{3} & 0 & 36 / t^{4} & -18 / t^{4} & 0 \\
-3 / t^{3} & 6 / t^{3} & 0 & -18 / t^{4} & 36 / t^{4} & 0 \\
0 & 0 & 18 / t^{3} & 0 & 0 & 108 / t^{4}
\end{array}\right]
\end{aligned}
$$

and

$$
\mathbf{T}_{b o t}=\left[\begin{array}{cccccc}
1 / t^{2} & -1 / 2 t^{2} & 0 & 6 / t^{3} & 3 / t^{3} & 0 \\
-1 / 2 t^{2} & 1 / t^{2} & 0 & 3 / t^{3} & -6 / t^{3} & 0 \\
0 & 0 & 3 / t^{2} & 0 & 0 & -18 / t^{3} \\
-6 / t^{3} & 3 / t^{3} & 0 & 36 / t^{4} & -18 / t^{4} & 0 \\
3 / t^{3} & -6 / t^{3} & 0 & -18 / t^{4} & 36 / t^{4} & 0 \\
0 & 0 & -18 / t^{3} & 0 & 0 & 108 / t^{4}
\end{array}\right] \text { (9) }
$$

\section{Fully Stress Design (FSD) Theory}

We will apply the FSD on a thin shell finite element. The thickness of element $t_{i}$ will be considered to be a design variable and the iterative relation will be given by $[1,2]$

$$
t_{i}^{(k+1)}=\frac{\sigma_{i e q}^{(k)}}{\sigma_{L}} \cdot t_{i}^{(k)}
$$

where $\sigma_{i e q}$ is von Mises equivalent stress for the shell element. It is convenient to use, for example, the procedure based on a discrete distribution of interval of admissible values of design variable (e.g. interval partition method) and subsequent evaluation of $\sigma_{i e q}$ for each $t_{i}$. So, then the nearest smaller value of calculated equivalent stress with respect to $\sigma_{L}$ will determine the new value of $t_{i}$.

The geometric interpretation of the method is shown in Fig. 1. Starting from the similarity of triangles $\mathrm{OAB}$ and $\mathrm{OCD}$ we will get

$$
\frac{\sigma_{i e q}^{(k)}}{\left(\frac{1}{t_{i}}\right)^{(k)}}=\frac{\sigma_{L}}{\left(\frac{1}{t_{i}}\right)^{(k+1)}}
$$




\section{caMMNICOIIONS}

From this equation we obtain the well-known iterative relation (10). The mentioned geometric interpretation shows that the new estimation $t_{i}^{(k+1)}$ is approximated from points $[0,0]$ and

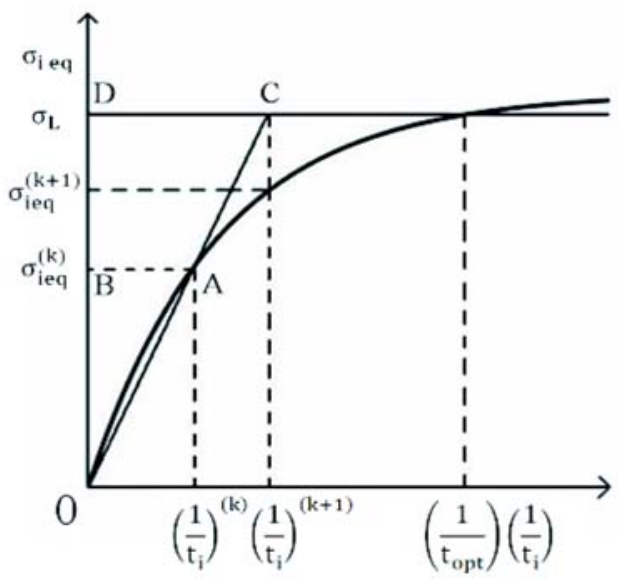

Fig. 1 Geometric interpretation of "classic" FSD

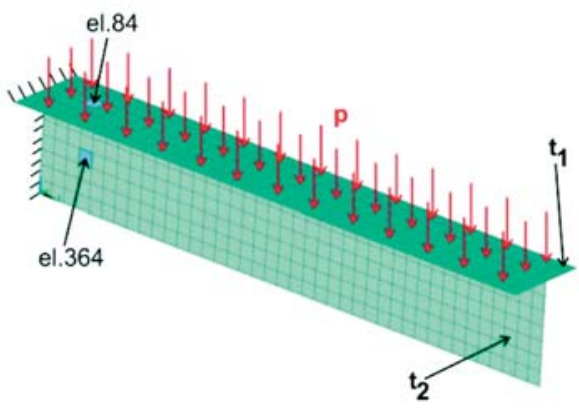

Fig. 3 One-sided fixed testing beam $p=0.05 \mathrm{MPa}$

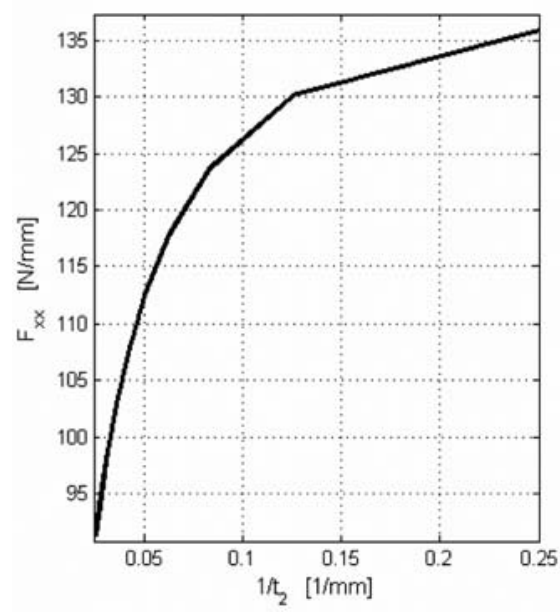

Fig. 5 Relation $F_{x x}-t_{2}$ for element 84, for $t_{l}=20 \mathrm{~mm}$ - one-sided fixed beam $\left[\left(1 / t_{i}\right)^{(k)}, \sigma_{L}\right]$. From the numerical mathematical point of view we are speaking about Regula Falsi method (method of chordssecants).

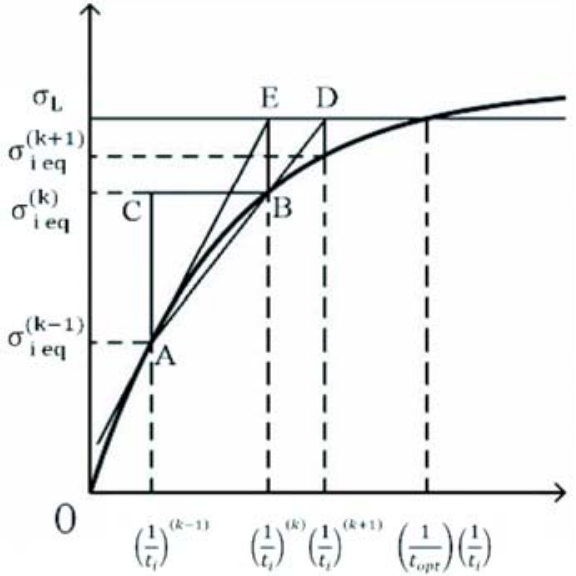

Fig. 2 Geometric interpretation of "new" FSD

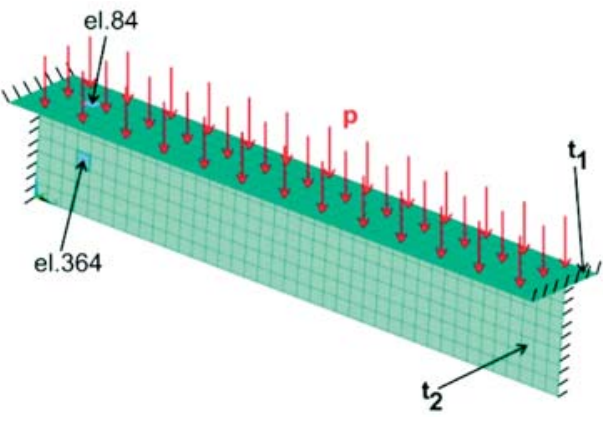

Fig. 4 Two-sided fixed testing beam $p=0.05 \mathrm{MPa}$

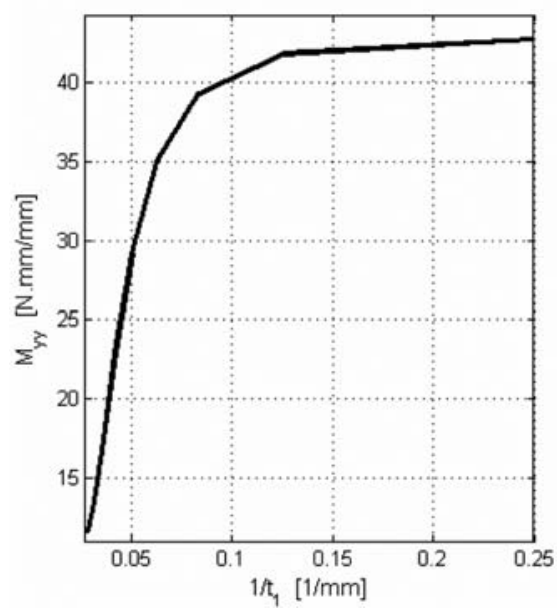

Fig. 6 Relation $M_{y y}-t_{1}$ for element 84 , for $t_{2}=20 \mathrm{~mm}-\mathrm{two}$-sided fixed beam 
On the basis of experiences with classical relation (10), the authors proposed two new computational algorithms. The first one arises from the idea of approximation of new solution from the previous two solutions which leads to the method which is very similar to Newton's tangent method. Geometric interpretation of the method is shown in Fig. 2. Starting from the similarity of triangles $\mathrm{ABC}$ and $\mathrm{BDE}$ we will get

$$
\frac{\sigma_{i e q}^{(k)}-\sigma_{i e q}^{(k-1)}}{\left(\frac{1}{t_{i}}\right)^{(k)}-\left(\frac{1}{t_{i}}\right)^{(k-1)}}=\frac{\sigma_{L}-\sigma_{i e q}^{(k)}}{\left(\frac{1}{t_{i}}\right)^{(k+1)}-\left(\frac{1}{t_{i}}\right)^{(k)}}
$$

and after modification we obtain the following iterative relation

$$
\left(\frac{1}{t_{i}}\right)^{(k+1)}=\left(\frac{1}{t_{i}}\right)^{(k)}+\frac{\sigma_{L}-\sigma_{i e q}^{(k)}}{\sigma_{i e q}^{(k)}-\sigma_{i e q}^{(k-1)}}\left[\left(\frac{1}{t_{i}}\right)^{(k)}-\left(\frac{1}{t_{i}}\right)^{(k-1)}\right]
$$

New estimation $\left(1 / t_{i}\right)^{(k+1)}$ results from the approximation of points $\left[\left(1 / t_{i}\right)^{(k-1)}, \sigma_{i e q}^{(k-1)}\right]$ and $\left[\left(1 / t_{i}\right)^{(k)}, \sigma_{\text {ieq }}^{(k)}\right]$. The introduced computational procedure is applicable also for truss, beam and shell finite elements.

The second proposed algorithm is suitable especially for shell finite elements.

It arises from the numerical study of effect of element thickness change on internal forces and moments [1]. The numerical analyses (tests) series was realized and it clearly proved the propriety of approximation of internal forces (moments). Two examples were tested (Figs. 3, 4). The dependence of force (moment) vs. element thickness is illustrated in Figs. $(5,6)$.

The numerical tests showed that the assumption of constant internal forces and moments per unit length $F_{x x}, F_{y y}, F_{x y}, M_{x x}, M_{y y}$, $M_{x y}$ in thin shells can cause troubles with convergence of solution and algorithms efficiency. This problem can be eliminated by the linear approximation of dependence between the mentioned internal forces per unit length and inverse value of element thickness $t_{i}$ (more details in e.g. [1] and Fig. 7). Next, we will assume that for $t_{i} \rightarrow \infty$ expression $\left(1 / t_{i}\right) \rightarrow 0$ and an internal force (moment) per unit length $T \rightarrow 0$. So, then the final approximation relation will be given by

$$
T_{\text {aprox }}^{(k+1)}=\frac{t^{2}}{t^{(k+1)}} \cdot T^{k} \quad \text { or } \quad \mathbf{f}_{\text {aprox }}^{(k+1)}=\frac{t^{k}}{t^{(k+1)}} \cdot \mathbf{f}^{k},
$$

where $k$ is the number of iteration step, in which we find a new value of $t^{(k+1)}$ from discrete design variables interval, for which we will predict internal force quantities per unit length $\mathbf{f}_{\text {aprox }}^{(k+1)}=$ $=\left[F_{x x}, F_{y y}, F_{x y}, M_{x x}, M_{y y}, M_{x y}\right]^{T}$ and von Mises stress $\sigma_{e q}$. Vector $f^{k}$ is the actual vector of internal forces and moments determined from FEM analysis in $k$-th iteration step, $t^{k}$ is the value of design variable, for which FEM analysis in $k$-th iteration step was performed. Using (5) and (6) we can calculate the approximated value of equivalent stress in $(k+1)$-th iteration step as follows

$$
\begin{aligned}
& \left(\left.\sigma_{\text {eq }}^{2}\right|_{\text {top }}\right)_{\text {aprox }}^{(k+1)}=\left(\mathbf{f}_{\text {aprox }}^{(k+1)}\right) \cdot \mathbf{T}_{\text {top }}^{(k+1)} \cdot \mathbf{f}_{\text {aprox }}^{(k+1)}=\left(\mathbf{f}^{k}\right)^{T} \cdot \frac{t^{k}}{t^{(k+1)}} \cdot \\
& \cdot \mathbf{T}_{\text {top }}^{(k+1)} \cdot \frac{t^{k}}{t^{(k+1)}} \cdot \mathbf{f}^{k}
\end{aligned}
$$

or

$$
\begin{aligned}
& \left(\left.\sigma_{e q}^{2}\right|_{b o t}\right)_{a p r o x}^{(k+1)}=\left(\mathbf{f}_{a p r o x}^{(k+1)}\right) \cdot \mathbf{T}_{b o t}^{(k+1)} \cdot \mathbf{f}_{a p r o x}^{(k+1)}=\left(\mathbf{f}^{k}\right)^{T} \cdot \frac{t^{k}}{t^{(k+1)}} . \\
& \cdot \mathbf{T}_{b o t}^{(k+1)} \cdot \frac{t^{k}}{t^{(k+1)}} \cdot \mathbf{f}^{k} .
\end{aligned}
$$

The mentioned approximation can decrease the number of iteration steps.

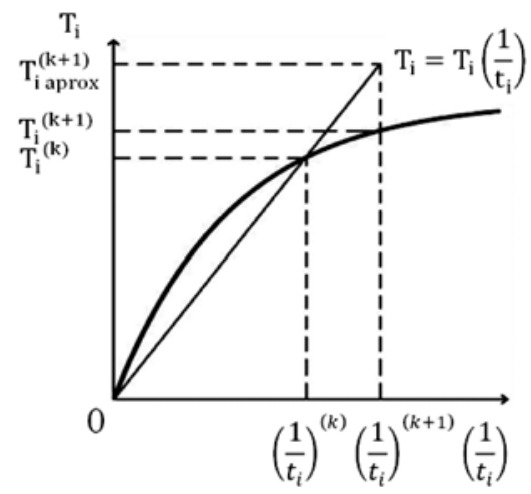

Fig. 7 Principle of the linear approximation of $T(1 / t)$

\section{Comparison Study of the Proposed Algorithms}

The presented computational algorithms were tested and compared on a cranked beam (Fig. 8). Four-node thin shell isoparametric finite elements were used for this testing mechanical problem. The number of elements was 5462 and number of nodes was 5739 . The material parameters used: Young's modulus $E=2.1 \cdot 10^{5} \mathrm{MPa}$ and Poisson's ratio $\vartheta=0.3$. The boundary conditions were defined as follows

- forces $=>$ pressure $p=0.05 \mathrm{MPa}$ (Fig. 8, magenta part),

- displacements $=>$ zero displacement on marked areas (Fig. 8, green part).

Three optimizing variables were selected for the process of optimization - thicknesses of flanges and web (See Fig. 9 - blue, green and red parts). The maximum design stress considered $\sigma_{L}=$ $=120 \mathrm{MPa}$. Vector $t_{\text {start }}=[40,40,40] \mathrm{mm}$ was suggested as the start point and discrete design variables were chosen from interval $\langle 8,40\rangle \mathrm{mm}$ with increment of $1 \mathrm{~mm}$. The optimizing process was terminated when the following convergence conditions were fulfilled

- stress convergence condition

$$
\left|\frac{\sigma_{L}-\sigma_{i}}{\sigma_{L}}\right| \leq 0.2 ; \sigma_{L}-\sigma_{i}>0,
$$

- design variable convergence condition

$$
\frac{t_{i}^{(k)}-t_{i}^{(k+1)}}{t_{i}^{(k)}} \leq 0.05 ; \sigma_{L}-\sigma_{i}>0 .
$$




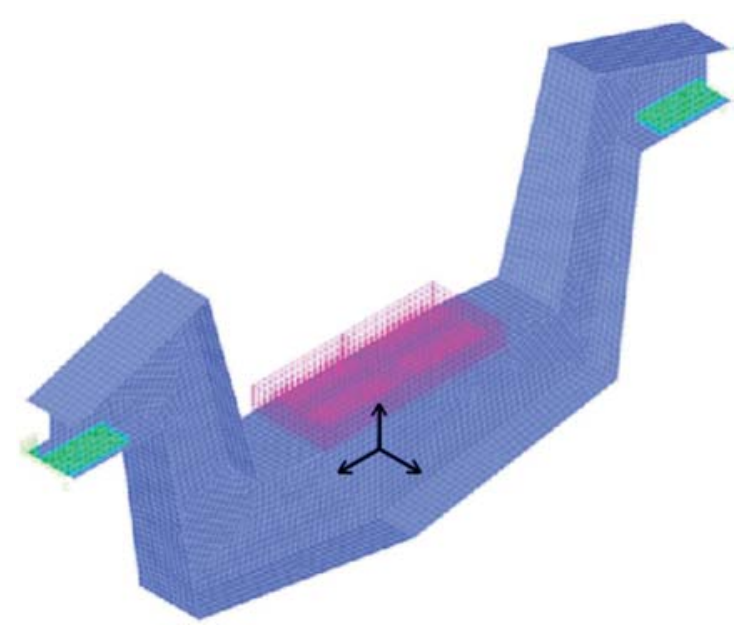

Fig. 8 Force and displacement boundary conditions

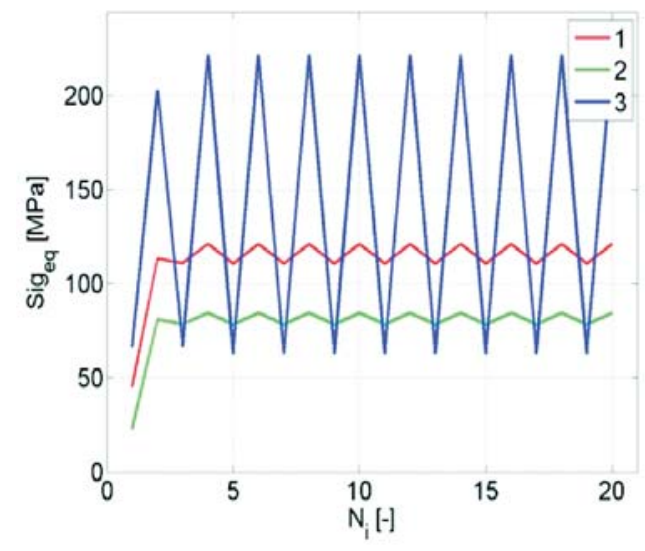

Fig. 10 History of stress convergence for $1^{\text {st }}$ algorithm

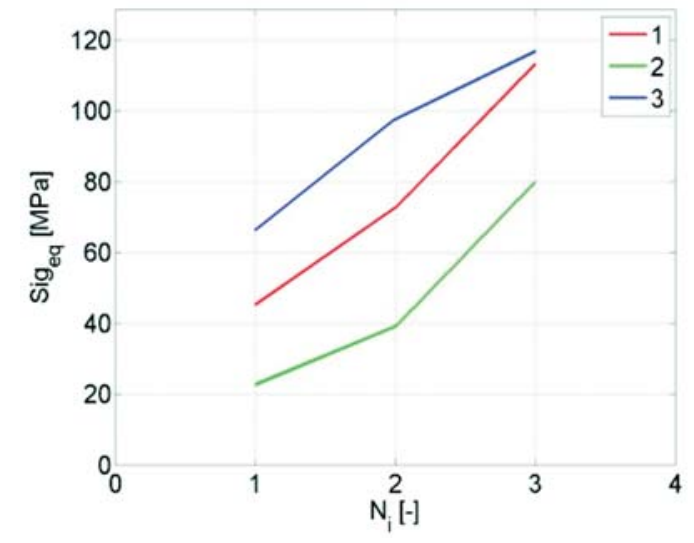

Fig. 12 History of stress convergence for $2^{\text {nd }}$ algorithm

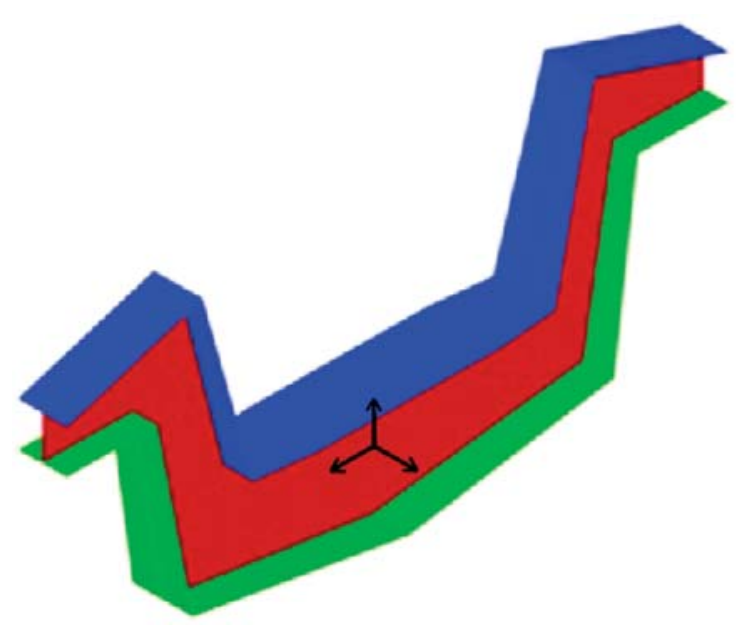

Fig. 9 Optimizing groups identification red $-1^{\text {st }}$ design variable green $-2^{\text {nd }}$ design variable blue - $3^{\text {rd }}$ design variable

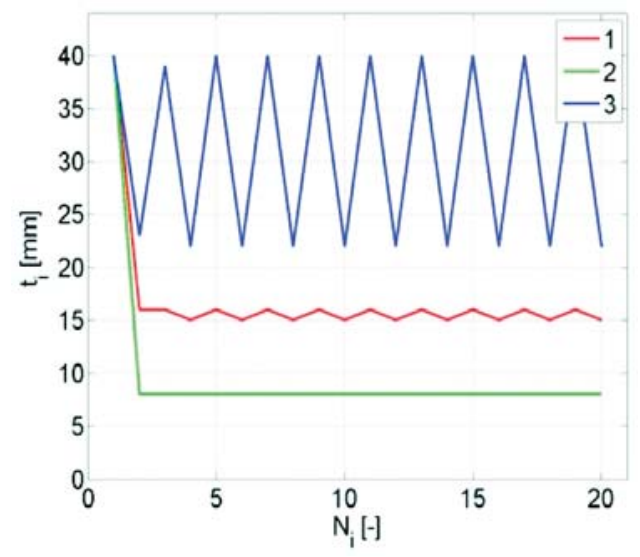

Fig. 11 History of thickness convergence for $1^{\text {st }}$ algorithm

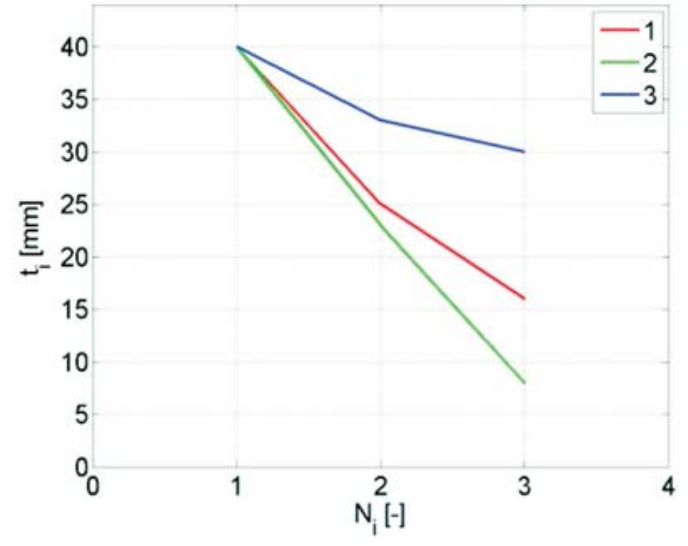

Fig. 13 History of thickness convergence for $2^{\text {nd }}$ algorithm

36 - COMMUNICATIONS $3 / 2012$ 


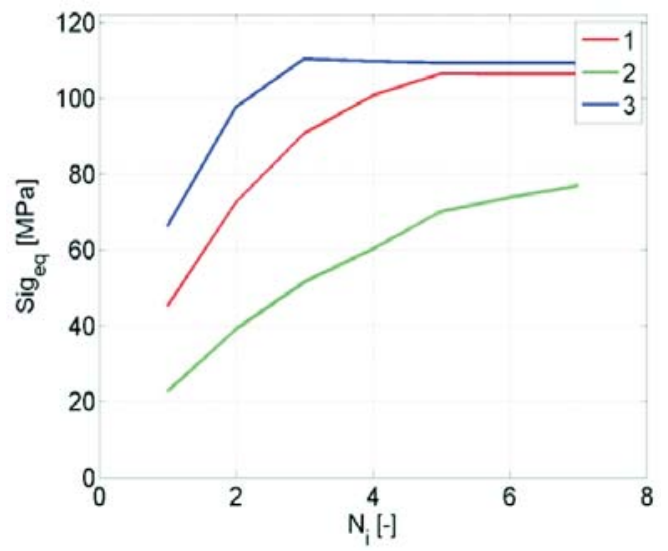

Fig. 14 History of stress convergence for $3^{\text {rd }}$ algorithm

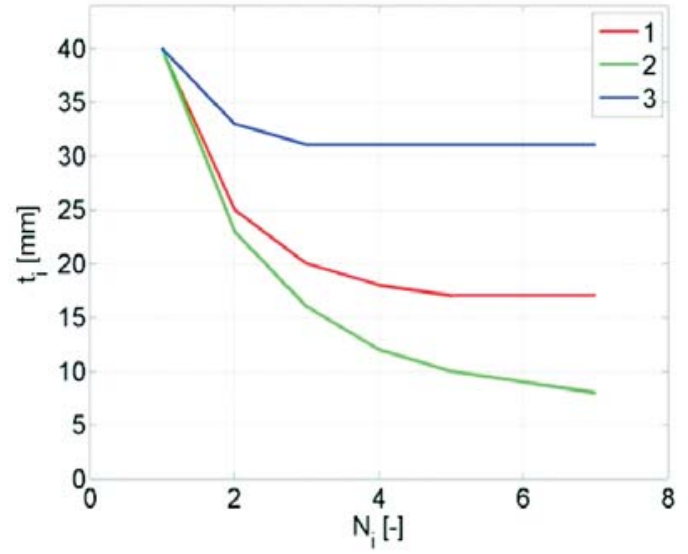

Fig. 15 History of thickness convergence for $3^{\text {rd }}$ algorithm
The results of solution are summarized into Tables 1, 2 and Figures 10-15. Graphic presentation shows that classic FSD algorithm does not have to converge necessarily and in the case of the shell computational model the discrete optimization can be inconvenient. Other two presented algorithms have perspective and they converge very well. For problems with lower number of optimizing variables it is better to apply algorithm No. 2. Experiences of the authors indicate that the third algorithm is more suitable for problems with a higher number of optimizing variables (more than 10).

Table 1

\begin{tabular}{|c|c|c|c|c|c|c|c|}
\hline \multicolumn{10}{|c|}{ Algorithm no. 2 } \\
\hline $\begin{array}{c}\text { Iter. } \\
\text { No. }\end{array}$ & $\begin{array}{c}t_{1} \\
{[\mathrm{~mm}]}\end{array}$ & $\begin{array}{c}t_{2} \\
{[\mathrm{~mm}]}\end{array}$ & $\begin{array}{c}t_{3} \\
{[\mathrm{~mm}]}\end{array}$ & $\begin{array}{c}\text { Stress_1 } \\
{[\mathrm{MPa}]}\end{array}$ & $\begin{array}{c}\text { Stress_2 } \\
{[\mathrm{MPa}]}\end{array}$ & $\begin{array}{c}\text { Stress_3 } \\
{[\mathrm{MPa}]}\end{array}$ & $\begin{array}{c}\text { Weight } \\
{[\mathrm{kg}]}\end{array}$ \\
\hline 1 & 40 & 40 & 40 & 45.2 & 22.6 & 66.2 & 660 \\
\hline 2 & 25 & 23 & 33 & 72.6 & 39.0 & 97.7 & 441 \\
\hline 3 & 16 & 8 & 30 & 113.2 & 79.8 & 116.8 & 291 \\
\hline
\end{tabular}

\section{Conclusion}

Our paper deals with the theoretical aspects and numerical realization of three fully stress optimizing algorithms focusing on shell finite elements models. The presented computational procedures were inbuilt into MATLAB's software module MAT_FSD which cooperates with FE software ADINA. Testing examples support the authors' considerations about the effectiveness of the proposed method.
Table 2

\begin{tabular}{|c|c|c|c|c|c|c|c|}
\hline \multicolumn{10}{|c|}{ Algorithm no. 3 } \\
\hline $\begin{array}{c}\text { Iter. } \\
\text { No. }\end{array}$ & $\begin{array}{c}t_{1} \\
{[\mathrm{~mm}]}\end{array}$ & $\begin{array}{c}t_{2} \\
{[\mathrm{~mm}]}\end{array}$ & $\begin{array}{c}t_{3} \\
{[\mathrm{~mm}]}\end{array}$ & $\begin{array}{c}\text { Stress_1 } \\
{[\mathrm{MPa}]}\end{array}$ & $\begin{array}{c}\text { Stress_2 } \\
{[\mathrm{MPa}]}\end{array}$ & $\begin{array}{c}\text { Stress_3 } \\
{[\mathrm{MPa}]}\end{array}$ & $\begin{array}{c}\text { Weight } \\
{[\mathrm{kg}]}\end{array}$ \\
\hline 1 & 40 & 40 & 40 & 45.1 & 22.6 & 66.2 & 660 \\
\hline 2 & 25 & 23 & 33 & 72.6 & 39.0 & 97.7 & 441 \\
\hline 3 & 20 & 16 & 31 & 90.7 & 51.5 & 110.1 & 363 \\
\hline 4 & 18 & 12 & 31 & 100.7 & 60.2 & 109.8 & 329 \\
\hline 5 & 17 & 10 & 31 & 106.5 & 70.2 & 109.4 & 313 \\
\hline 6 & 17 & 9 & 31 & 106.4 & 73.9 & 109.3 & 308 \\
\hline 7 & 16 & 8 & 30 & 113.2 & 79.8 & 116.8 & 291 \\
\hline
\end{tabular}

The significant result of the presented study is mainly the fact that using of classical fully stress design method for shell structures modelled by means of the finite element method is inconvenient. The authors proposed two new algorithms which have overpassed this problem. These methods converge well, they are effective in the number of iteration steps and they have big perspective for large optimizing problems where the goal is to find hundreds of structural parameters by application of relatively low number of iteration steps.

\section{Acknowledgements}

This work has been supported by VEGA grant No. $1 / 0125 / 09$.

\section{References}

[1] SAGA, M., VASKO, M., KOCUR, R., TOTH, L., KOHAR, R.: Application of Optimizing Algorithms in Solid Mechanics (in Slovak), VTS ZU Zilina, 2006. 


\section{COMMNICOIIONS}

[2] HAFTKA, R. T., GURDAL, Z.: Elements of Structural Optimization. Kluwer Academic Publisher, 1992.

[3] DEKYS, V., SAPIETOVA, A., KOCUR, R.: On the Reliability Estimation of the Conveyer Mechanism using the Monte Carlo Method. Proc. COSIM2006, Krynica-Zdroj, august 2006, pp. 67-74.

[4] JAKUBOVICOVA L., KOPAS P., HANDRIK M., VASKO M.: Computational and Experimental Analysis of Torsion and Bending Loading of Specimen. In-Tech 2010, Prague, pp. 395-400.

[5] LEITNER B., KOPAS P.: The Vector Autoregressive Moving Average Models Asa Tool for Stochastically Loaded Dynamic Systems Identification. Machine Dynamics Research, 2010, No. 4, vol. 34, pp. 32-41.

[6] ZIENKIEWICZ, O. C.: The Finite Element Method in Engineering Science. McGraw, Hill, New York, 1971.

[7] ZMINDAK, M., NOVAK, P.: Particles Interactions in Composites Reinforced by Fibre and Spherical Inclusions. Communications - Scientific Letters of the University of Zilina, No. 2, 2009.

[8] ZMINDAK, M., RIECKY, D.: SOUKUP, J.: Failure of Composites with Short Fibers. Communications - Scientific Letters of the University of Zilina, No. 4, 2010.

[9] SAGA, M., VASKO, M.: Stress Sensitivity Analysis of the Beam and Shell Finite Elements. Communications - Scientific Letters of the University of Zilina, vol. 11, No. 2, 2009, pp. 5-12.

[10] KWON, Y. W., BANG, H.: The Finite Element Method using MATLAB. CRC Press University of Minnesota, 1996.

[11] BATHE, K. J.: Finite Element Procedures. New Persey : Prentice Hall, 1996.

[12] SAPIETOVA, A., DEKYS, V., VASKO, M.: A Numerical ModelingRotating Machine Having Unbalance and the Measuring of its Dynamical Properties, Metalurgija (Metallurgy), No. 2, vol. 49, 2010, pp. 503-507. 